This item was submitted to Loughborough's Research Repository by the author.

Items in Figshare are protected by copyright, with all rights reserved, unless otherwise indicated.

\title{
Unmanned aerial vehicle-based hazardous materials response: Information- theoretic hazardous source search and reconstruction
}

PLEASE CITE THE PUBLISHED VERSION

https://doi.org/10.1109/MRA.2019.2943006

\section{PUBLISHER}

Institute of Electrical and Electronics Engineers

VERSION

AM (Accepted Manuscript)

\section{PUBLISHER STATEMENT}

(c) 2019 IEEE. Personal use of this material is permitted. Permission from IEEE must be obtained for all other uses, in any current or future media, including reprinting/republishing this material for advertising or promotional purposes, creating new collective works, for resale or redistribution to servers or lists, or reuse of any copyrighted component of this work in other works.

\section{LICENCE}

CC BY-NC-ND 4.0

\section{REPOSITORY RECORD}

Hutchinson, Michael, Cunjia Liu, Paul Thomas, and Wen-Hua Chen. 2019. "Unmanned Aerial Vehicle-based Hazardous Materials Response: Information-theoretic Hazardous Source Search and Reconstruction". Loughborough University. https://hdl.handle.net/2134/9873602.v2. 


\title{
UAV Based HAZMAT Response: Information-theoretic Hazardous Source Search and Reconstruction
}

\author{
Michael Hutchinson, Cunjia Liu, Member, IEEE, Paul Thomas, and Wen-Hua Chen, Fellow, IEEE
}

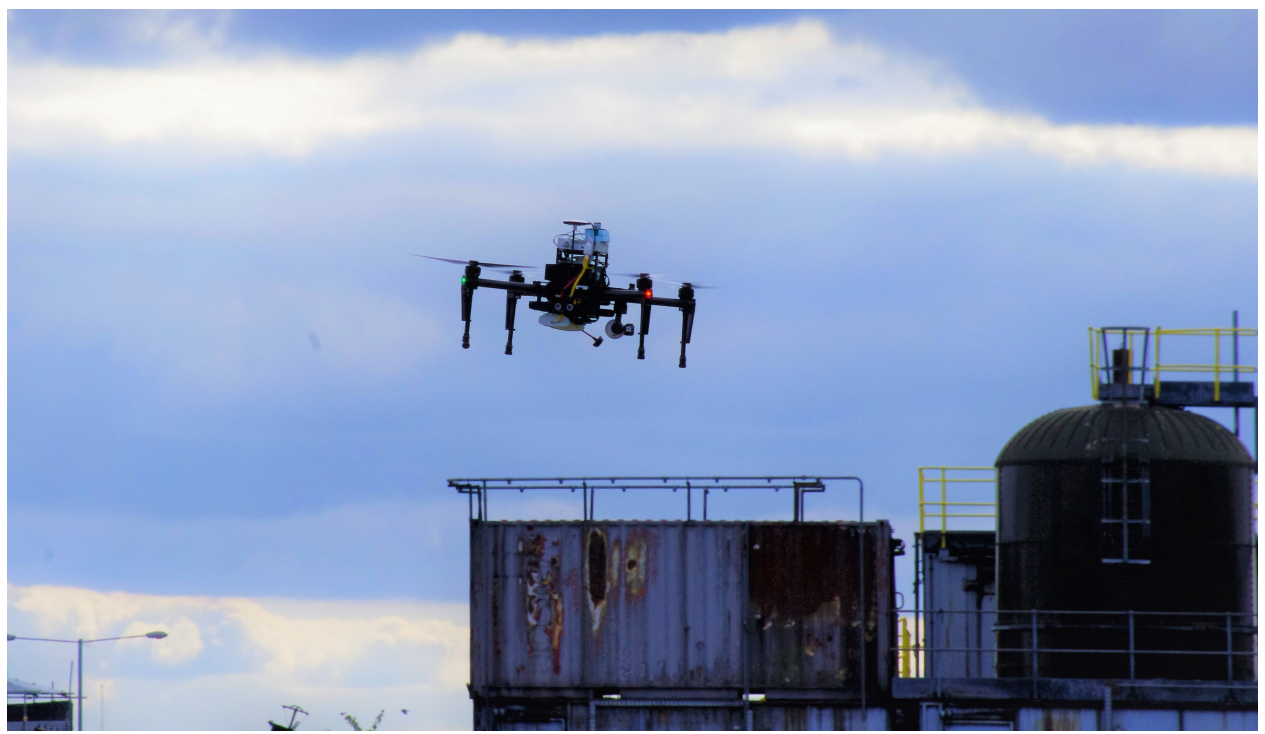

$\mathbf{H}$ AZARDOUS material (HAZMAT) released into the atmosphere poses both an immediate and chronic risk to human health. Characteristic examples include the Sarin gas terrorist attacks in Japan (1995), the infamous chemical accidents of Bhopal, India (1984) and Seveso, Italy (1976), Nuclear disasters such as Fukushima (2012), and the recent use of chemical weapons and nerve agents in Syria (2013-2018). A prompt and accurate prediction of the whereabouts of the hazardous material and a forecast of its dispersion and deposition is important to enable responders to undertake appropriate mitigation strategies and extract people from affected regions. Hazard predictions, however, require accurate knowledge of the release parameters (the so-called source term), as well as the local meteorological information. In many situations this information is unknown, or highly uncertain. HAZMAT sensor readings will indicate the presence of HAZMAT and this must be turned rapidly into a warning in order to ensure the safety of personnel in the vicinity. This currently requires either a static network of pre-deployed sensors, which can be costly and necessitate substantial planning, or the manual collection of sensor measurements, e.g., using handheld devices and HAZMAT suits, which can be time consuming and places personnel at risk.

Michael Hutchinson, Cunjia Liu and Wen-Hua Chen are with the Department of Aeronautical and Automotive Engineering, Loughborough University, UK, e-mail: \{M.Hutchinson2, C.Liu5, W.Chen\}@lboro.ac.uk.

Paul Thomas is with the Defence Science and Technology Laboratory (Dstl), Porton Down, Salisbury, UK, email: PATHOMAS@mail.dstl.gov.uk

Manuscript received April 30, 2019.
The optimal response to releases of HAZMAT would be stand-off, rapid, and reliable; keeping personnel out of danger, whilst maximising the efficiency of the response. We propose an autonomous UAV with integrated HAZMAT sensors as a system providing: a reconnaissance and survey capability for obtaining real-time, targeted (i.e., most informative) HAZMAT measurements without endangering personnel. This article presents our recent research and experiments undertaken to develop such a system.

It is most common to perform source term estimation (STE) using a network of static detectors on the ground [1]. However, the focus of this article is on estimating the source term of a release using a UAV equipped with an in-situ atmospheric concentration sensor. An unmanned aerial system (UAS) can supplement an existing network, or replace it entirely, by intelligently gathering spatio-temporally indexed concentration measurements of the release. There is limited research in the area of STE using a UAS, however, it has recently been validated in an experimental study previously conducted by the authors [2]. In this study a systematic flight pattern was executed to gather the HAZMAT data which were used in a Bayesian inference based estimation algorithm to estimate the source parameters; taking into consideration the large amount of uncertainty in the measurements from the sensor and the atmospheric dispersion process.

It is hypothesised that a more accurate and rapid response could be achieved by planning the path of the UAS on-line in response to the information gained during the mission. This is termed an information theoretic approach, which was shown 
in [3] to outperform a systematic approach for source term estimation in simulation. The ability to estimate the source term of a dispersive release using an information theoretic approach was recently explored in [4] where simple experiments validated the approach with a ground robot equipped with a metal oxide (MOX) sensor in an indoor arena with fans to create a wind field and smoke to simulate a HAZMAT release. Experiments in more realistic natural environments are limited. Most related to this article is the previous work conducted by the authors [2]. A UAV executed a pre defined flight path to collect measurements from the onboard atmospheric sensor which were used to estimate the source term of an atmospheric release using a sequential Monte Carlo algorithm. The paper introduced a likelihood function to relate the noisy measurements from the UAV, exacerbated by the short sampling/averaging times used to take measurements, with a simple Atmospheric Transport and Dispersion (ATD) model; this enabled the source term of a diffusive release to be estimated in realistic outdoor conditions.

This article presents an extendible framework for information theoretic hazardous source search and reconstruction followed by an experimental assessment of the system and then realistic demonstrations conjured by potential end users from the emergency services. The novelty of the work is in the integration of a recently proposed Bayesian STE algorithm with an information theoretic planner, and a simplistic outline of a framework for information theoretic source reconstruction, where each component can be extended to improve the overall system. In addition, the experimental assessment of the information theoretic search algorithm using a UAV is the first of its kind, paving the way to a deployable system given some further improvements to a few of the components. The results show a significant reduction in search time of using the proposed information theoretic planner when compared to that of a pre-planned uniform flight path [2]. Finally, the value of the system is demonstrated in realistic scenarios in addition to further demonstrations of the inference engine using a manual and a preplanned flight for data collection.

The remainder of the article is organised as follows. Firstly, related work is reviewed in Section I. An overview of the proposed response system is provided in Section II. In Section III, the inference engine for STE is described. The information theoretic path planner is described in Section IV. Section V describes the results of experiments conducted to verify the method and a comparison with a more conventional pre planned search. Demonstrations of the system in trials setup by potential end users are described in Section VI followed by a discussion of the results. Finally, conclusions are given in Section VII.

\section{RELATED WORK}

There are many potential responses to releases of airborne HAZMAT. Two prevailing methods are mapping of the hazard distribution [5]-[7] and localisation of the source [8]. The focus of this article, however, is on source reconstruction, otherwise known as source term estimation, the discussion on related work is therefore limited accordingly.
The source term of a release includes its location and emission rate, in addition to other variables needed to model the spread of the HAZMAT using an ATD model. The task is most commonly formulated as an inverse problem and approached using a network of static atmospheric concentration and meteorological sensors. Bayesian inference and optimisation algorithms have both been proposed to estimate the source term given the measurement data [1], along with alternative methods, e.g., neural network [9] and approximate Bayesian computation [10]. Other sources of measurement data have been seen in literature such as the use of toxic health effects information [11].

Another option is to mount the atmospheric concentration sensors on mobile platforms, which in turn necessitates a sensor planning function. Such a planning function has been demonstrated in [12] to estimate the source terms of radiation releases using multiple robots. In other areas, methods known as information theoretic planning, information based planning, active information acquisition, active sensing, or on-line informative path planning, have been successfully applied problems such as simultaneous localisation and mapping (SLAM), known as active SLAM [13], target search, localisation and tracking [14], [15], source localisation [16] and 3D mapping [17].

A closely related work explored source localisation of a atmospheric release, not STE, using a UAV incorporating various reactive algorithms [18]. This differs from the current work as this paper proposes to use an information theoretic approach, and estimates not only the source location but other useful parameters of the source term such as the emission rate.

\section{THE SYSTEM}

Locating a source undergoing atmospheric dispersion is challenging. Atmospheric turbulence and a weak source lead to noisy and sporadic measurements which makes infeasible the intuitive approaches of localising an emitting source by following the positive concentration gradient [19]. Living organisms that are known to adopt such a gradient based, or "chemotaxis" approach, are of a microscopic scale such as Escherichia Coli bacteria [20]. On a larger scale the approach is unsuccessful.

This paper presents an information theoretic source search and reconstruction framework to overcome this challenge, in conjunction with experiments to validate the extendible framework in its current state of development. By virtue of the Bayesian inference framework the presented system is extendible to cater for more scenarios and to increase the robustness of the overall system. The framework, outlined in Fig. 1, consists predominantly of two parts: an inference engine, and a path planner.

The inference engine is based on Bayes' theorem which allows the parameters of the source to be estimated despite the noisy measurements from the atmospheric concentration sensor, uncertainties in the meteorological conditions, and errors and assumptions in the underlying models. The output of the inference is a set of probability density functions (pdfs) that represent what is known about the source term 


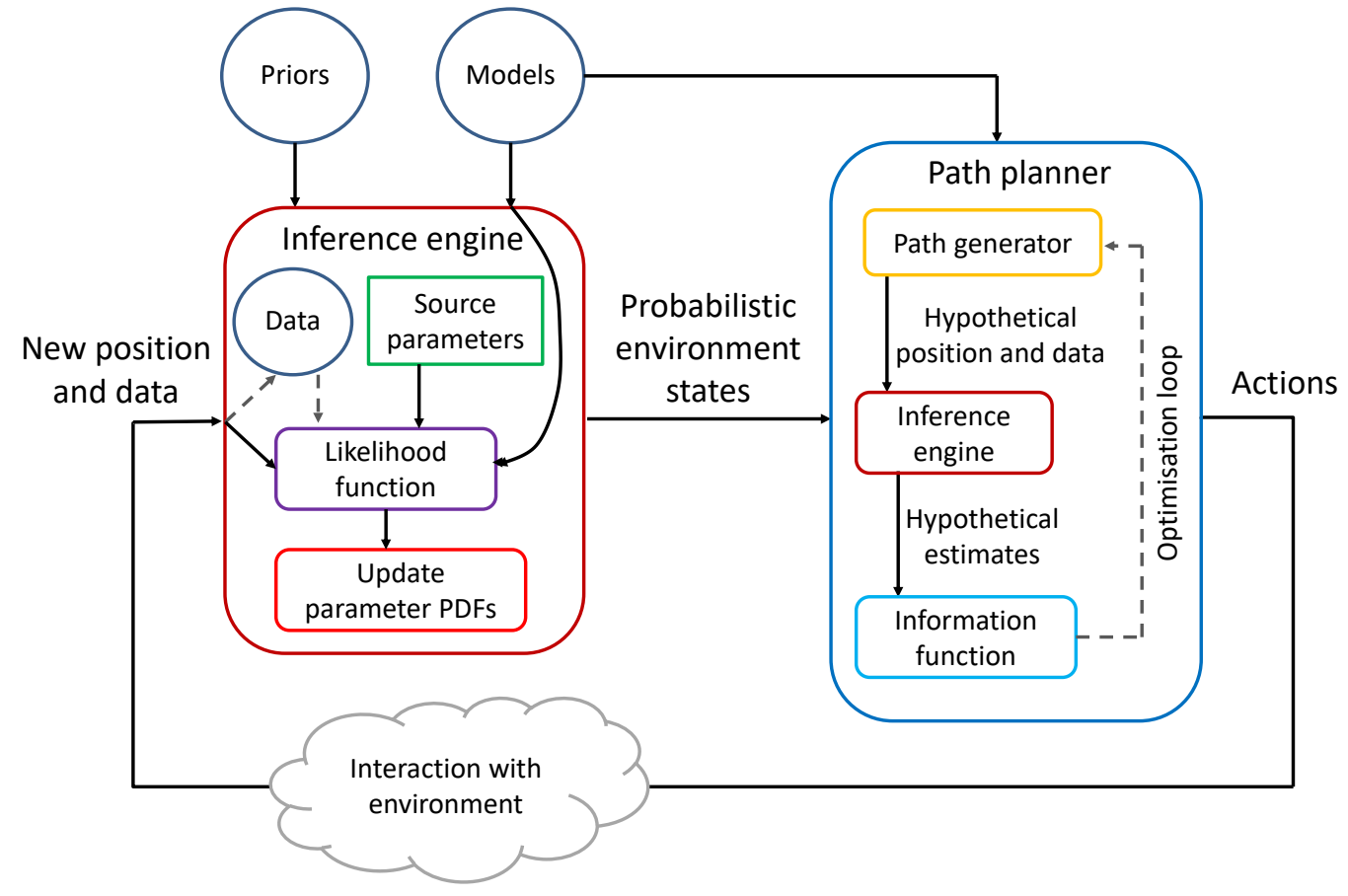

Fig. 1: Information-theoretic source search and reconstruction framework.

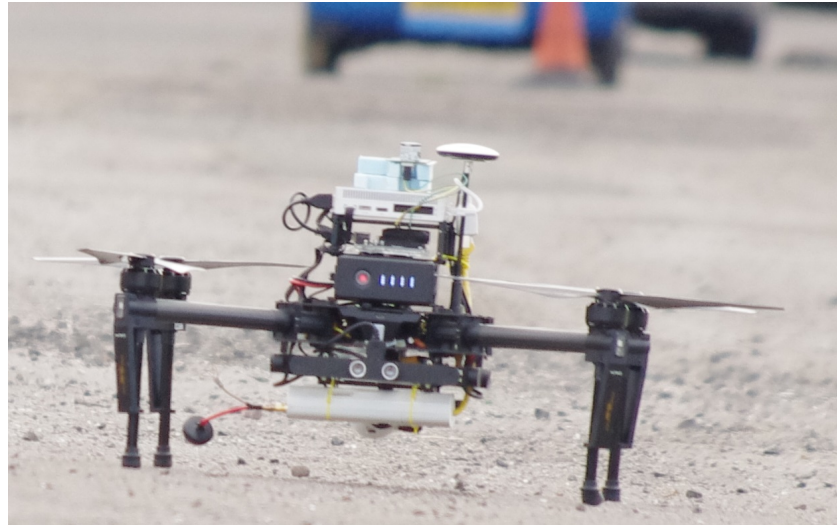

Fig. 2: UAV system.

parameters. This provides valuable information such as the location of the source and its rate of emission which provides all the information needed to forecast the dispersion of the HAZMAT from its source. The inference engine can be used independently of the information theoretic planner, fed using data from pre-planned flights, manual flights, or even static detectors on the ground. The focus of this article is on the information theoretic planner, nonetheless, a manual and a pre-planned flight is also demonstrated in Section VI. The inference engine is described further in Section III.

The information theoretic planner selects the best path to follow by considering the predicted information gain from the subsequent measurements. The planner uses the current pdfs of the source parameters and an atmospheric transport and dispersion model to predict measurements at the proposed new measurement positions. The new measurements are then input into the inference engine which outputs new pdfs of the source parameters assuming the potential measurements were true. The information gain is then calculated which can be done using various measures of information such as the KullbackLeibler divergence. An optional optimisation loop can be used to find the most informative path. The information theoretic path planner is described further in Section IV.

It should be noted that the path planner can be replaced by an operator guided flight mode in the scenarios where human operators may have a better understanding of the scene. This flight mode includes the manual flight and predefined search pattern using waypoints.

The physical system consists primarily of a quadrotor UAV (DJI Matrice 100) and a laptop as a base station. The quadrotor is equipped with a Photoionisation Detector (PID) to measure the atmospheric concentration of HAZMAT, shown mounted on the top of the platform in Fig. 2. The measured concentrations are sent to a ground station laptop which runs the Bayesian estimation of the source parameters and sends the next position demand to the UAV, based on the information theoretic reward. There is the potential to run the algorithms using the onboard computer of the UAV, however, during the experimental trials a ground station laptop was used for simplicity, to enable more seamless algorithm development, and for visualisation purposes during demonstrations to potential end users. The Robot Operating System (ROS) was used for all communications between the autopilot and the on board computer, and likewise between the onboard computer and the ground-station. 


\section{INFERENCE ENGINE}

The inference engine is based upon the Bayesian framework, which allows uncertainties in measurements, input variables, and models to be accounted for using an appropriate probability distribution. The inference engine takes as an input the source parameters $\Theta$ and their initial prior distributions $\pi\left(\Theta_{0}\right) \equiv p\left(\Theta_{0}\right)$. Measurement data $\mathbf{z}_{1: k}$, in the form of concentration measurements from the HAZMAT sensor (and potentially also meteorological measurements) collected at sequential time instants from 1 to $k$, are then used to recursively update the estimates according to Bayes' rule, such that

$$
p\left(\Theta_{k} \mid \mathbf{z}_{1: k}\right)=\frac{p\left(z_{k} \mid \Theta_{k}\right) p\left(\Theta_{k} \mid \mathbf{z}_{1: k-1}\right)}{p\left(z_{k} \mid \mathbf{z}_{1: k-1}\right)} .
$$

where $\mathbf{z}_{1: k}$ implies that the measurement data are collected at the corresponding locations $\mathbf{p}_{1: k}$. The current state of knowledge regarding the source parameters, or in general parameters that represent the environment of interest, can then be represented by a posterior pdf $p\left(\Theta_{k} \mid \mathbf{z}_{1: k}\right)$.

\section{A. Source parameters and dispersion model}

In this work, the source term contains the necessary information that characterises a source and its dispersion in conjunction with an ATD model. The ATD model will in turn provide the expected concentration at position $\mathbf{p}_{k}$ produced from a hypothesised source with parameters $\Theta_{k}$, denoted as $\mathcal{M}\left(\mathbf{p}_{k}, \Theta_{k}\right)$. Any relevant model can be used; there exist highly complex particle tracking models, Computational Fluid Dynamics (CFD) techniques [21], [22], or equations derived from analytical solutions to the advection-diffusion equations [19]. The model and its corresponding source parameters are interchangeable without any other changes to the algorithm, and in practice would be a trade-off between the computational capabilities of the processor and the time requirements for the prediction, with consideration of the likely scenarios the system would operate in.

In this work, a model derived from analytical solutions to the advection diffusion equation given various assumptions is used, which shall be referred to the Isotropic plume (IP) model as verified in [2]. The model is fast running and based on the assumption of a steady state plume with a consistent mean wind velocity, source strength, and turbulent conditions.

In this model, the expected concentration to be read by a detector at position $\mathbf{p}_{k}=\left[\begin{array}{lll}p_{x, k} & p_{y, k} & p_{z, k}\end{array}\right]^{\top}$ from a source at position $\mathbf{p}_{s}=\left[\begin{array}{lll}x_{s} & y_{s} & z_{s}\end{array}\right]^{\top}$, releasing airborne HAZMAT at a rate of $q_{s}$ with average lifetime $\zeta_{s 2}$ in an environment with mean wind speed $u_{s}$, wind direction $\phi_{s}$ and diffusivity $\zeta_{s 1}$ is given by:

$$
\begin{gathered}
\mathcal{M}\left(\mathbf{p}_{k}, \Theta_{k}\right)=\frac{q_{s}}{4 \pi \zeta_{s 1}\left\|\mathbf{p}_{k}-\mathbf{p}_{s}\right\|} \exp \left[\frac{-\left\|\mathbf{p}_{k}-\mathbf{p}_{s}\right\|}{\lambda}\right] \times \\
\exp \left[\frac{-\left(x_{k}-x_{s}\right) u_{s} \cos \phi_{s}}{2 \zeta_{s 1}}\right] \exp \left[\frac{-\left(y_{k}-y_{s}\right) u_{s} \sin \phi_{s}}{2 \zeta_{s 1}}\right],
\end{gathered}
$$

where $\lambda=\sqrt{\frac{\zeta_{s 1} \zeta_{s 2}}{1+\left(u_{s}^{2} \zeta_{s 2}\right) /\left(4 \zeta_{s 1}\right)}}$. Some of the parameters of the source term could be assumed known, leaving only the unknown, or uncertain parameters in need of estimation. To account for uncertainties all the parameters of the ATD model used in this article are considered uncertain. Hence, the parameter vector of the source term can be defined as: $\Theta_{k}=\left[\begin{array}{llllll}\mathbf{p}_{s}^{\top} & q_{s} & u_{s} & \phi_{s} & \zeta_{s 1} & \zeta_{s 2}\end{array}\right]^{\top}$.

\section{B. Atmospheric sensing model and likelihood function}

To construct the likelihood function $p\left(z_{k+1} \mid \Theta_{k+1}\right)$ used in Eq. (1), there must be a method of linking HAZMAT sensor measurements $z_{k}$ with the expected observations $\mathcal{M}\left(\mathbf{p}_{k}, \Theta_{k}\right)$ from the model, leading to an atmospheric sensing model.

The sensing model features detection events, where measurements from the HAZMAT detector picked up some concentrations from the source, and non-detection events, where the measurement did not surpass a pre-specified threshold $z_{t h r}$. The threshold is set high enough to minimise false detections, whilst maintaining sufficient sensitivity. The observational data, and subsequently the likelihood function, can be split among these detections $z_{k}=\bar{z}_{k}$ and non-detections $z_{k}=\underline{z}_{k}$, such that

$$
p\left(z_{k} \mid \Theta_{k}\right)= \begin{cases}p\left(\bar{z}_{k} \mid \Theta_{k}\right), & \text { if } z_{k} \geq z_{t h r} \\ p\left(\underline{z}_{k} \mid \Theta_{k}\right), & \text { if } z_{k}<z_{t h r}\end{cases}
$$

The observational model linking detection data $\bar{z}_{k}$ with the source term parameters $\Theta_{k}$ is given as $\bar{z}_{k}=\mathcal{M}\left(\mathbf{p}_{k}, \Theta_{k}\right)+\bar{v}_{k}$, where $\bar{v}_{k}$ encapsulates the various errors between the measured and modelled concentration at a particular position $\mathbf{p}_{k}$. The discrepancy can arise from measurement error, input error, model error and stochastic uncertainty [23], which is suggested to be modelled as a Gaussian distribution [24]. Thus, the likelihood function for a detection event is defined as $p\left(\bar{z}_{k} \mid \Theta_{k}\right)=\mathcal{N}\left(\bar{z}_{k} ; \mathcal{M}\left(\mathbf{p}_{k}, \Theta_{k}\right), \sigma_{k}^{2}\right)$, where the variance $\sigma_{k}^{2}$ is a function of the modelled concentration such that $\sigma_{k}^{2} \propto \mathcal{M}\left(\mathbf{p}_{k}, \Theta_{k}\right)$.

A non-detection event on the other hand, can be caused by three hypothesised scenarios which are assumed to be independent: a) the concentration measurement is only a result of background and instrument noise, denoted by $\mathcal{E}_{b}$; b) the non detection is a result of intermittency caused by turbulence or a missed detection, denoted by $\mathcal{E}_{m}$, typically exacerbated by the short sampling intervals of the UAV; or, c) the concentration includes contributions from both the source and background, denoted by $\mathcal{E}_{s}$, although it did not amount to a value above the concentration threshold. Combining the three hypotheses and further treating $\underline{z}_{k}$ as an interval measurement result in the following likelihood of a non-detection:

$$
\begin{aligned}
& p\left(\underline{z}_{k} \mid \Theta_{k}\right)=P_{b} \cdot p\left(\underline{z}_{k} \mid \mathcal{E}_{b}\right)+P_{m}+P_{s} \cdot p\left(\underline{z}_{k} \mid \mathcal{E}_{s}, \Theta_{k}\right) \\
& =P_{b} \cdot \Phi\left(z_{t h r} ; \mu_{b}, \sigma_{b}^{2}\right)+P_{m}+P_{s} \cdot \Phi\left(z_{t h r} ; \mathcal{M}\left(\mathbf{p}_{k}, \Theta_{k}\right), \sigma_{k}^{2}\right)
\end{aligned}
$$

where $P_{b}, P_{m}$ and $P_{s}$ represent the probabilities of events $\mathcal{E}_{b}$, $\mathcal{E}_{m}$ and $\mathcal{E}_{s}$, respectively, $\mu_{b}$ and $\sigma_{b}^{2}$ are the mean and variance of the background noise and $\Phi(\cdot ; a, b)$ denotes the cumulative distribution function of a normal distribution with mean $a$ and variance $b . P_{b}, P_{m}$ and $P_{s}$ are tuning parameters and their value should be set during the experiments and satisfy $P_{b}+$ $P_{m}+P_{s}=1$. 


\section{Implementation}

Given the appropriate models and Bayesian formulations, the source term estimation algorithm is implemented using a particle filter. The output is an approximation of the posterior distribution $p\left(\Theta_{k} \mid \mathbf{z}_{1: k}\right)$, which represents the current state of knowledge about the source parameters. In particle filter, the posterior distribution from Eq. (1) is approximated by a set of weighted random samples $\left\{\Theta_{k}^{(i)}, w_{k}^{(i)}\right\}_{i=1}^{N}$ such that

$$
p\left(\Theta_{k} \mid \mathbf{z}_{1: k}\right) \approx \sum_{i=1}^{N} w_{k}^{(i)} \delta\left(\Theta_{k}-\Theta_{k}^{(i)}\right),
$$

where $\delta(\cdot)$ is a Dirac delta function, $\Theta_{k}^{(i)}$ is a sample representing a potential source term realisation and $w_{k}^{(i)}$ is the corresponding normalised weighting such that $\sum_{i=1}^{N} w_{k}^{(i)}=1$.

The process of recursively calculating the posterior distribution at sampling instance $k$ is summarised in Algorithm 1 and more details can be found in [2].

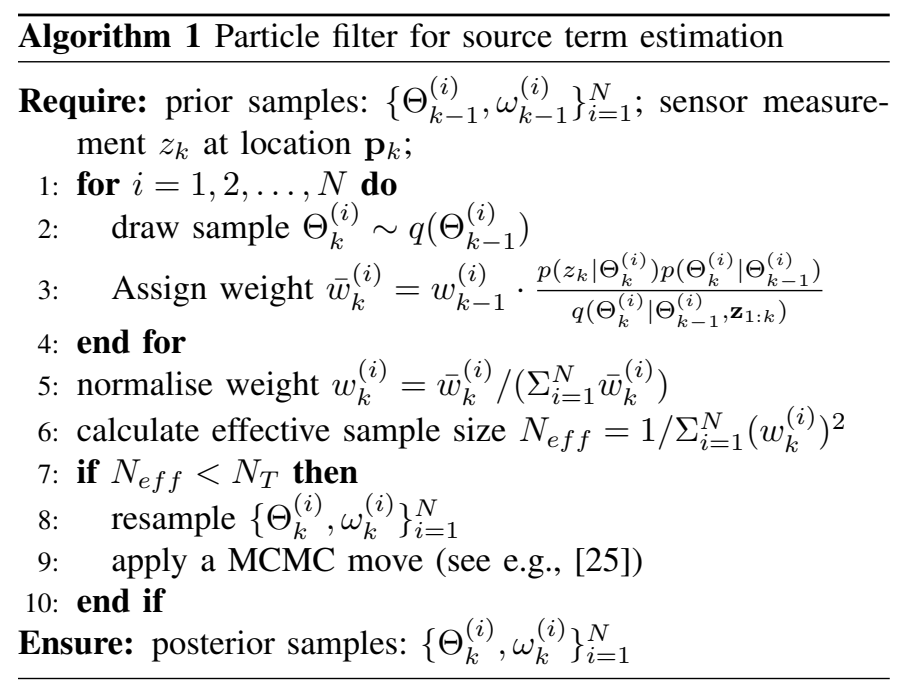

\section{PATH PLANNER}

\section{A. Information-theoretic search}

The goal of sensor planning is to choose the manoeuvre $\mathbf{a}_{k}^{*}$ from an admissible set of actions $\Psi_{k}=\{\uparrow, \downarrow, \leftarrow, \rightarrow, \odot, \times\}$, that is expected to be the most informative. Here, the arrows refer to moves in the upwind, downwind and left/right crosswind directions, and $(\odot, \times)$ refer to an increase or reduction in UAV altitude. The reward function for sensor planning is inspired by the literature on optimal experiment design [26], where it is referred to as the utility function $\Upsilon\left(z_{k+1}\left(\mathbf{a}_{k}\right)\right)$. This is used to capture the information gain on the estimate of $\Theta_{k}$ given the next sensor data $z_{k+1}$ after taking the action $\mathbf{a}_{k}$. Different utility functions can be adopted. Since the future measurement $z_{k+1}$ is generally unknown, it is suggested that the optimal design of an experiment should be the one that maximises the expected utility of the subsequent measurement $\mathbb{E}\left[\Upsilon\left(\hat{z}_{k+1}\left(\mathbf{a}_{k}\right)\right)\right]$, where the expectation is calculated with respect to the hypothetical future measurement $\hat{z}_{k+1}$. The experimental design problem is adapted to direct a mobile sensor, where the choice of the next experiment is synonymous with the movement of the sensor. The maximization problem can be written as:

$$
\mathbf{a}_{k}^{*}=\arg \max _{\mathbf{a}_{k} \in \Psi} \mathbb{E}\left[\Upsilon\left(\hat{z}_{k+1}\left(\mathbf{a}_{k}\right)\right)\right] .
$$

The expected utility of manoeuvre $\mathbf{a}_{k}$ can be further expressed as an integral based on the probability of a future measurement $\hat{z}_{k+1}\left(\mathbf{a}_{k}\right)$ and its corresponding utility $\Upsilon\left(\hat{z}_{k+1}\left(\mathbf{a}_{k}\right)\right)$ :

$$
\begin{aligned}
& \mathbb{E}\left[\Upsilon\left(\hat{z}_{k+1}\left(\mathbf{a}_{k}\right)\right)\right] \\
& =\int_{\hat{z}_{k+1} \in \mathcal{Z}} p\left(\hat{z}_{k+1}\left(\mathbf{a}_{k}\right) \mid \mathbf{z}_{1: k}\right) \Upsilon\left(\hat{z}_{k+1}\left(\mathbf{a}_{k}\right)\right) \mathrm{d} \hat{z}_{k+1},
\end{aligned}
$$

where $\mathcal{Z}$ is the range of the possible future measurement at the future sampling position. In this article, the utility of the manoeuvre $\mathbf{a}_{k}$ is defined as the Kullback-Leibler divergence between the predicted source term distributions before and after the measurement $\hat{z}_{k+1}\left(\mathbf{a}_{k}\right)$ being taken into account, i.e., between the distributions $p\left(\Theta_{k+1} \mid \mathbf{z}_{1: k}\right)$ and $p\left(\Theta_{k+1} \mid \mathbf{z}_{1: k}, \hat{z}_{k+1}\left(\mathbf{a}_{k}\right)\right)$. Thus, the utility function is defined as

$$
\begin{aligned}
\Upsilon\left(\mathbf{a}_{k}, \hat{z}_{k+1}\right) & =D_{K L}\left(p\left(\Theta_{k+1} \mid \mathbf{z}_{1: k}, \hat{z}_{k+1}\left(\mathbf{a}_{k}\right)\right)|| p\left(\Theta_{k+1} \mid \mathbf{z}_{1: k}\right)\right) \\
& =\int_{\Theta_{k+1}} p\left(\Theta_{k+1} \mid \mathbf{z}_{1: k}, \hat{z}_{k+1}\left(\mathbf{a}_{k}\right)\right) \\
& \times \ln \frac{p\left(\Theta_{k+1} \mid \mathbf{z}_{1: k}, \hat{z}_{k+1}\left(\mathbf{a}_{k}\right)\right)}{p\left(\Theta_{k+1} \mid \mathbf{z}_{1: k}\right)} \mathrm{d} \Theta_{k+1}
\end{aligned}
$$

The sensor control strategy provides a full search algorithm under a single framework, which provides balanced exploration and exploitation by adapting to the state of the posterior density estimates of the source parameters. This is characterised by more explorative behaviour when the posterior distributions have a wide spread and are uninformative, and exploitative behaviour, directed towards the source, as the posterior distributions become more informative. The approach naturally moves towards the source location, as the posterior estimate becomes more certain.

\section{B. Implementation}

To calculate the expected utility $\mathbb{E}\left[\Upsilon\left(\hat{z}_{k+1}\left(\mathbf{a}_{k}\right)\right)\right]$ in Eq. (7) using the Monte Carlo method, a set of possible future measurements $\hat{z}_{k+1} \in \mathcal{Z}$, at location $\mathbf{p}_{k+1}$ after taking action $\mathbf{a}_{k}$, needs to be first generated. This process is given in Algorithm 2.

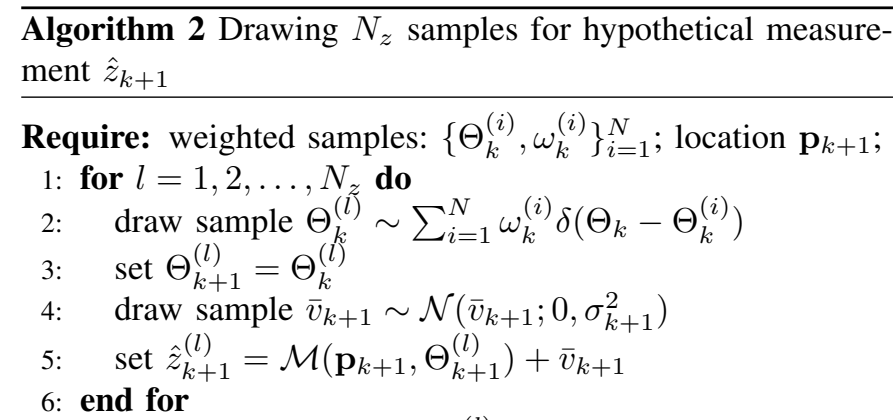

Ensure: weighted samples: $\left\{\hat{z}_{k+1}^{(l)}, \frac{1}{N_{z}}\right\}_{l=1}^{N_{z}}$ 
For each given hypothetical measurement $\hat{z}_{k+1}^{(l)}$, the utility function $\Upsilon(\cdot)$ defined in Eq. (8) can be evaluated. Based on the set of samples $\left\{\Theta_{k}^{(i)}, \omega_{k}^{(i)}\right\}_{i=1}^{N}$ resembling $p\left(\Theta_{k} \mid \mathbf{z}_{1: k}\right)$, the posterior distribution $p\left(\hat{\Theta}_{k+1}^{(l)} \mid \mathbf{z}_{1: k}, \hat{z}_{k+1}^{(l)}\right)$ can be approximated by a set of samples $\left\{\hat{w}_{k+1}^{(i, l)}, \hat{\Theta}_{k+1}^{(i, l)}\right\}_{i=1}^{N}$ using Algorithm 1 . Then, the corresponding utility function can be calculated by replacing the distributions in Eq. (8) with their respective sample approximations, such that

$$
\Upsilon\left(\hat{z}_{k+1}^{(l)}\left(\mathbf{a}_{k}\right)\right) \approx \sum_{i=1}^{N} \hat{w}_{k+1}^{(i, l)} \ln \frac{\hat{w}_{k+1}^{(i, l)}}{w_{k}^{(i)}} .
$$

At last, the expected utility function with respect to the hypothetical future measurements $\hat{z}_{k+1}^{(l)}, l=1, \ldots, N_{z}$ can be derived by taking average, such that

$$
\mathbb{E}\left[\Upsilon\left(\hat{z}_{k+1}\left(\mathbf{a}_{k}\right)\right)\right] \approx \frac{1}{N_{z}} \sum_{l=1}^{N_{z}} \sum_{i=1}^{N} \hat{w}_{k+1}^{(i, l)} \ln \frac{\hat{w}_{k+1}^{(i, l)}}{w_{k}^{(i)}} .
$$

The expected utility is calculated for all the manoeuvres in the set $\Psi$, then the UAV selects the move $\mathbf{a}_{k}^{*}$ that has the greatest expected utility. Following the manoeuvre, the robot takes a new observation $z_{k+1}$ and the estimation and sensor control cycle is iterated until some stopping criteria are reached. The action selection algorithm is summarised in Algorithm 3.

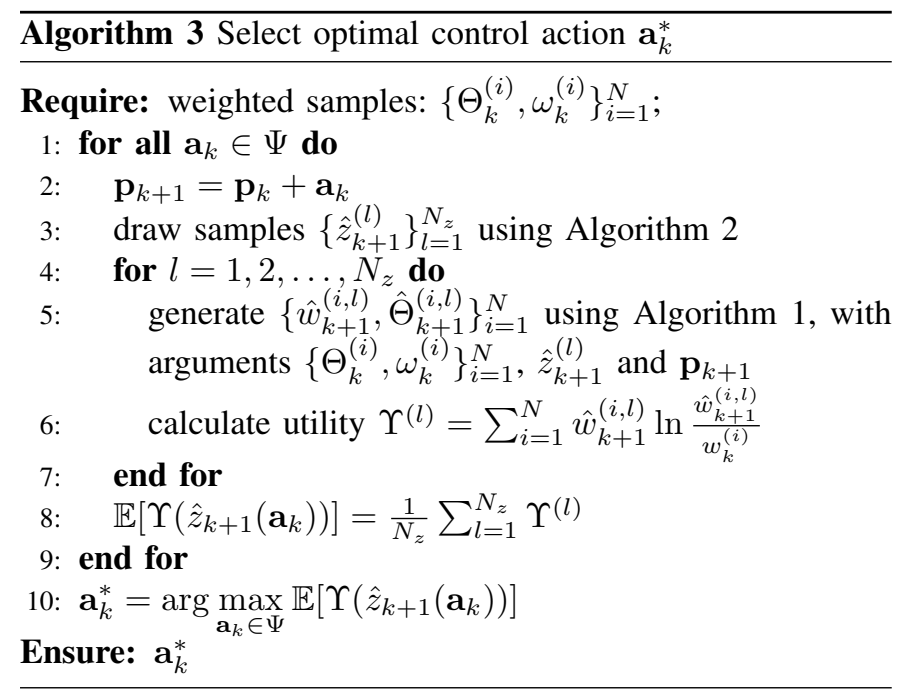

\section{EXPERIMENTS}

\section{A. Experiment set-up}

Experiments were performed to assess the performance of the information theoretic search and source reconstruction algorithm. The experiments were conducted outdoors in an uncontrolled open field, using the same setting as [2] in order to compare the information theoretic path planning strategy, presented in this article, with a uniform predefined path. The experiments used an acetone vapour source with a release/emission rate of approximately $1.5 \mathrm{~g} / \mathrm{s}$ and the UAS described in Section II.

The field used during the experiments was located near to Loughborough University, Leicestershire, UK. A large square within the field (approximately $40 \mathrm{~m}$ by $40 \mathrm{~m}$ ), containing the release, would represent the domain $\Omega$ which forms a part of the input to the algorithm. The remaining inputs are initial probability distributions that are required as prior information to the inference algorithm. They are as follows:

- The prior distributions for the location of the source $\left[p_{0}\left(x_{s}\right), p_{0}\left(y_{s}\right), p_{0}\left(z_{s}\right)\right]$ are set to uniform within the search area $\Omega$.

- A gamma distribution is used as the prior for the emission rate $p_{0}\left(q_{s}\right)=\mathcal{G}(1,5)$. This is a long tailed distribution to account for a large amount of uncertainty in the emission rate of the source. This prior was fixed during all of the experimental trials.

- The meteorological variables $\left[p_{0}\left(\phi_{s}\right), p_{0}\left(u_{s}\right)\right]$ are assigned with normal distributions $\mathcal{N}(\mu, \sigma)$ upon initialisation of the algorithm. In the future, the meteorological variables should be measured in-situ, onboard the UAV (as seen in [27]).

- The dispersion parameters $\left[\zeta_{s 1}, \zeta_{s 2}\right]$ were given uniform distributions with an appropriate range.

- The probabilities for non-detection events are set as $P_{b}=$ $0.1, P_{m}=0.2$ and $P_{s}=0.7$.

At each time step, the UAV would hover to take an averaged measurement from the onboard atmospheric sensor. The sample duration was set to 5 seconds. This was a short amount of time compared to source term estimation methods incorporating static sensors, where it is more common to sample for a few minutes. The 5 second sampling time was chosen as a trade-off between the measurement accuracy and search time. After the sample is collected and the source parameter estimates are updated, the UAV would proceed to the next measurement location defined by the information theoretic planning algorithm. The incremental step size between each measurement location was set to $4 \mathrm{~m}$.

\section{B. Illustrative run}

An illustrative run of an experiment, Trial 1, using the information theoretic planning algorithm is described in Fig. 3 and a video of Trial 9 is available in the supplementary materials. Overlaid on a map of the experimental field, the figure shows: the flight path of the UAV executing the information theoretic search at various snapshots in time; the measurements at each sampling location where the measured value is represented by the size of the white circles along the path; and the true position of the source. To begin the search, the system is initialised at discrete time step $k=0$ with the relevant prior information. The starting position of the UAV is indicated by the white square. The large number of red dots represents the random sample approximation used in the sequential Monte Carlo algorithm at the current time step (i.e., the current posterior distribution). At initialisation, in figure 3a, this is a uniform distribution within the search area. Each red dot represents a weighted source term realisation $\left\{\Theta_{k}^{(i)}, w_{k}^{(i)}\right\}$, where only the marginalised position estimates are visualised in the figure.

Figures 3a-3d show the path of the UAV and the measurement positions at various time steps. The figure demonstrates 


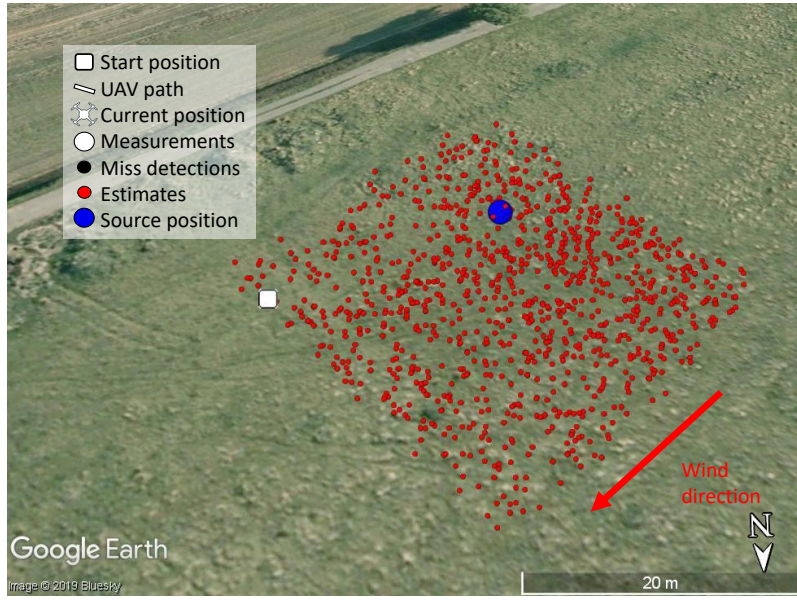

(a) Estimate at $\mathrm{k}=0$

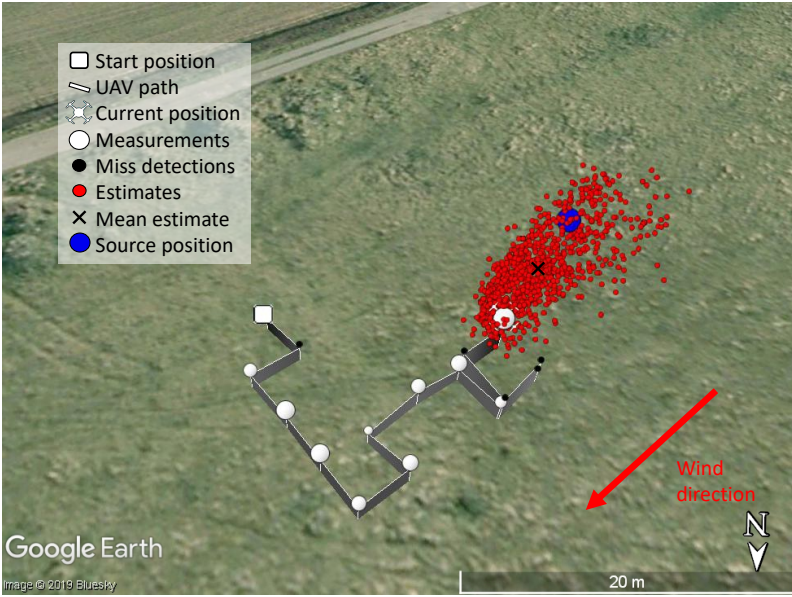

(c) Estimate at $\mathrm{k}=16$

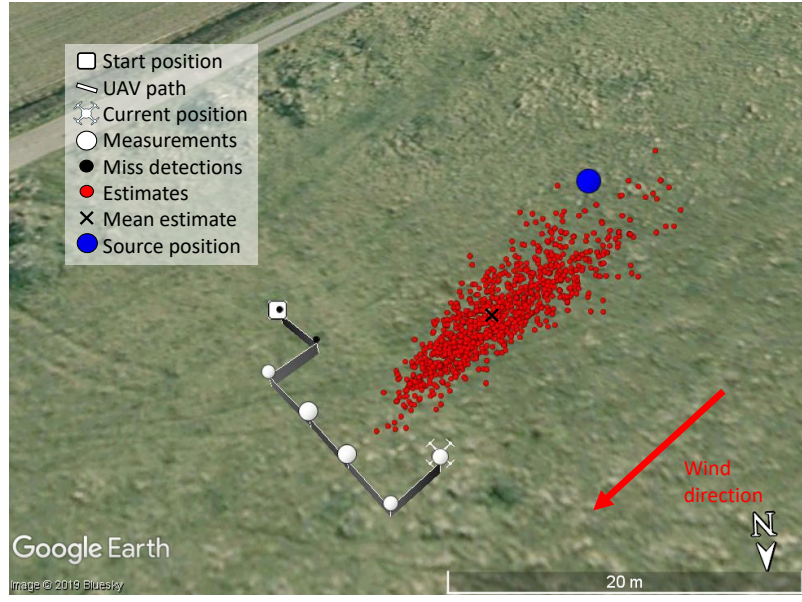

(b) Estimate at $\mathrm{k}=7$

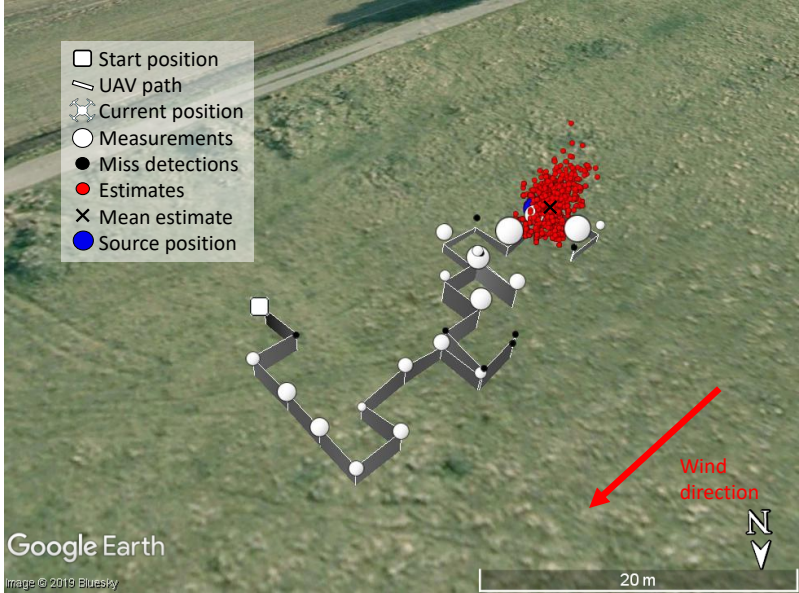

(d) Estimate at $\mathrm{k}=29$

Fig. 3: Example run of the information theoretic search algorithm at discrete time steps: (a) $k=0$ and (b) $k=7$ (c) $k=16$ and (d) $\mathrm{k}=29$.

how the information theoretic planner begins the search by moving in a crosswind direction, because this is the direction of maximum information gain. In response to positive detections from the PID detector the estimation algorithm is able to narrow down the location of the source in the crosswind direction as shown in Fig. 3b. At this point it begins to travel upwind towards the source. By time-step $k=29$, shown in Fig. 3d, the red dots have converged onto the location of the acetone vapour source, given by the blue circle. In this example the estimate of the location of the source was within $1.42 \mathrm{~m}$ of the true value. The emission rate estimate was reasonably accurate, but over predicted by 1.26 grams $/ \mathrm{s}$.

\section{Results}

The illustrative run and two other experiments are summarised in a condensed form in Fig. 4. The top figures show the resulting flight path (white line), wind direction (red arrow) and marginalised posterior estimate of the source location (heat map). The starting and ending positions of the UAV are given by the white square and diamond, respectively. The true position of the source is indicated by the black circle filled with a white cross and the algorithm's mean estimate is given by the hollow black circle. In a similar manner to the illustrative run, the information theoretic search algorithm guides the UAV crosswind until it begins to pick up positive measurements from the PID sensor. The final estimate, shown by the heat map, is accurate with more spread along the wind axis which is a common feature of source term estimation algorithms. The size of the white dots represent the measurements from the PID sensor. The challenge posed by large amounts of noise and intermittency in the sensor measurements is demonstrated in all of the upper figures of figure 4, where on several occasions there was no response from the PID detector downwind of the source. The bottom figures show the probability density estimate of the emission rate of the source, $p\left(q_{s} \mid \mathbf{z}_{1: k}\right)$. As shown in the figures, the true value of the emission rate typically falls within a standard deviation of the estimate.

The results of 10 trials using the information theoretic algorithm are summarised in Table I, including information about the source location estimates, emission estimates and some flight data. Overall, the Euclidean error is small given the scale of the experiments and, moreover, small given the severe amount of uncertainty in the dispersion process and even some uncertainty in the localisation of the UAV itself. 


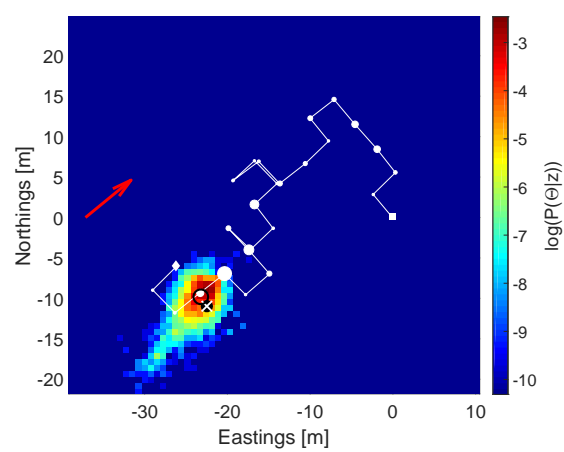

(a) Trial 1

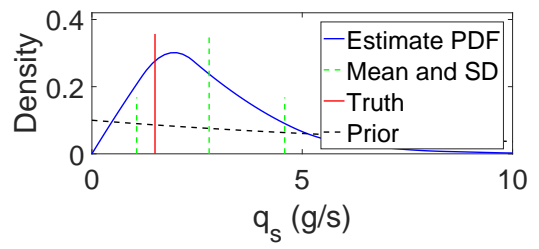

(d) Trial 1 emission estimate

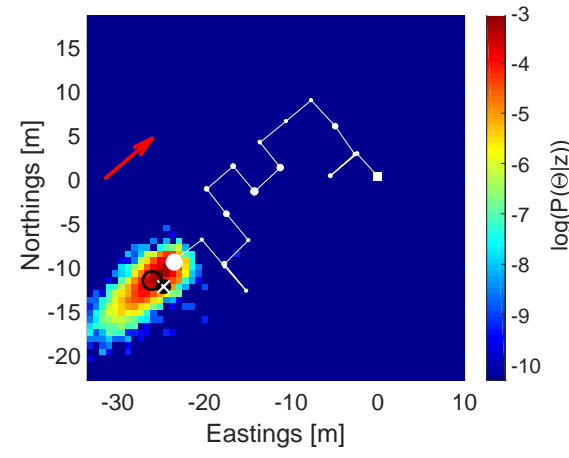

(b) Trial 2

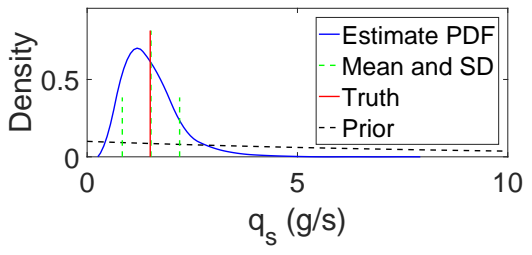

(e) Trial 2 emission estimate

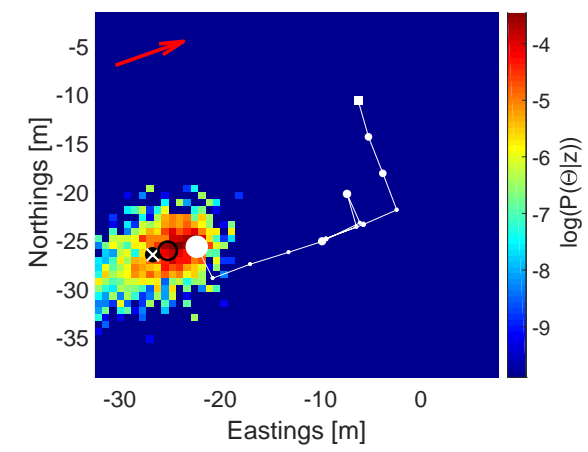

(c) Trial 5

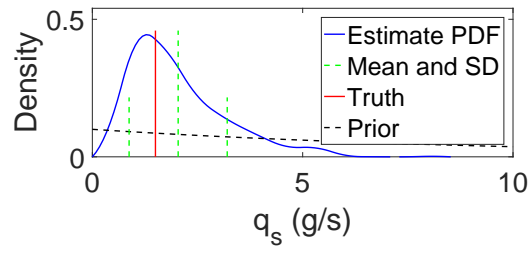

(f) Trial 5 emission estimate

Fig. 4: Summary of some information theoretic search experiments using the UAV. The heat map represents the marginalised posterior distribution for the source location estimate.

The emission estimates from the algorithm were encouraging, with errors typically within $1 \mathrm{~g} / \mathrm{s}$.

The results of the information theoretic experiments are compared with the uniform sweep results from the authors' previous work in [2]. The uniform sweep results are restricted to where the Isotropic plume model was used and where the scale of the experiments were similar to that used in the information theoretic trials. The average search time, the source position estimate RMSE, and the source emission rate RMSE using both methods are shown in Table II. Overall, in agreement with the simulations conducted in [3], the information theoretic search algorithm attained more accurate source position estimates and a significantly lower average search time. The emission rate RMSE was less affected; this is expected to be due to the large amount of noise in the sensor measurements for both strategies, and the lower number of measurements taken during the informative search before the source was found. It is expected that a longer sampling time, whilst taking atmospheric concentration measurements, would improve the emission rate estimate for both approaches, although penalising the search time.

\section{DEMONSTRATIONS}

The system was demonstrated in a full week of trials designed by first responders at the Fire Service College, UK. An overview of the trial environment is given in Fig. 5. There were two vignettes used to begin the trials: a suspected release of HAZMAT on an oil rig marked by the red box in Fig. 5a and a car crash with a suspected HAZMAT situation marked by the green box. The source used during the demonstrations was a cylinder of compressed propylene, shown on the oil rig in the top of Fig. 5b and near an overturned van in the figure underneath.
TABLE I: Summary of results for the information theoretic experimental trials, using the IP model, including the accuracy of the source location and emission rate estimates, and some flight data.

\begin{tabular}{|c|c|c|c|c|}
\hline \multicolumn{3}{|c|}{ Flight data } & \multicolumn{2}{|l|}{ Results } \\
\hline ID & $\begin{array}{l}\text { Time } \\
{[\mathrm{mm}: \mathrm{ss}]}\end{array}$ & $\begin{array}{l}\text { Wind speed } \\
{[\mathrm{m} / \mathrm{s}]}\end{array}$ & $\begin{array}{l}\text { Position } \\
\text { error }[\mathrm{m}]\end{array}$ & $\begin{array}{l}\text { Emission } \\
\text { error }[g / s]\end{array}$ \\
\hline 1 & $05: 31$ & 5 & 1.44 & 1.26 \\
\hline 2 & 04:05 & 6 & 1.22 & 0.09 \\
\hline 3 & $04: 48$ & 4 & 2.39 & 1.27 \\
\hline 4 & $05: 12$ & 3 & 7.04 & 0.16 \\
\hline 5 & 03:05 & 4 & 1.31 & 0.55 \\
\hline 6 & 06:02 & 3 & 1.76 & 1.09 \\
\hline 7 & $08: 28$ & 4 & 2.76 & 0.23 \\
\hline 8 & $06: 51$ & 5 & 2.08 & 0.63 \\
\hline 9 & $02: 37$ & 5 & 1.52 & 0.50 \\
\hline 10 & 07:01 & 4 & 3.81 & 0.73 \\
\hline
\end{tabular}

TABLE II: Accuracy of information theoretic search compared to sweep flight pattern

\begin{tabular}{llll}
\hline Method & $\begin{array}{l}\text { Average search } \\
\text { time [mm:ss] }\end{array}$ & $\begin{array}{l}\text { Position } \\
\text { RMSE [m] }\end{array}$ & $\begin{array}{l}\text { Emission } \\
\text { RMSE }[\mathrm{g} / \mathrm{s}]\end{array}$ \\
\hline $\begin{array}{l}\text { Sweep pattern } \\
\text { Information }\end{array}$ & $08: 05$ & 4.21 & 0.65 \\
theoretic search & $05: 22$ & 2.83 & 0.65 \\
\hline
\end{tabular}

The trials described in this article include: a manual flight around the oil rig, where measurements taken during the manual flight were used in the STE algorithm to estimate the location and emission rate of the release; an autonomous mission where the UAV followed a set of predefined waypoints to collect measurement data for the STE algorithm; and 


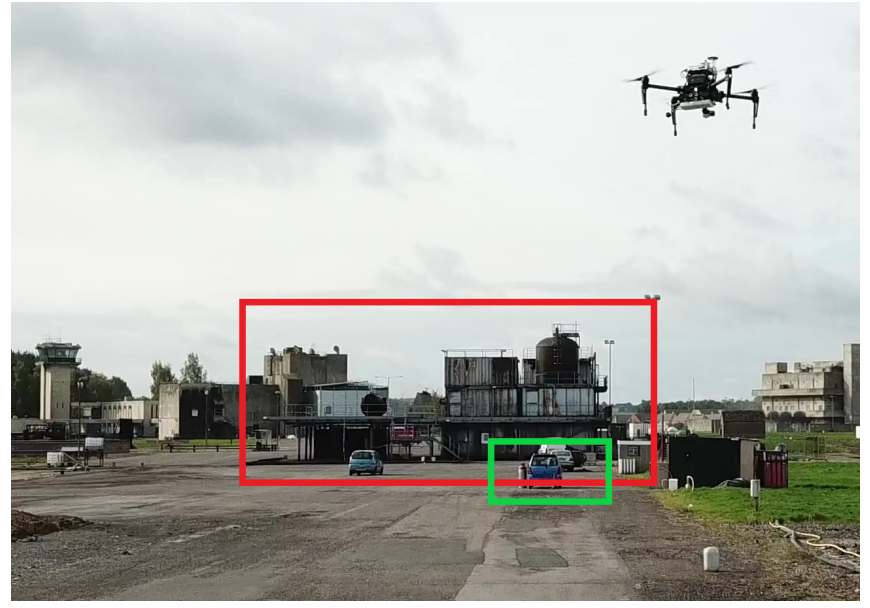

(a)

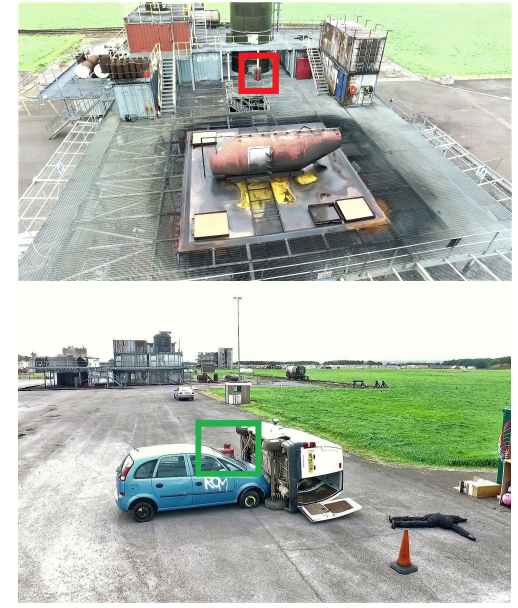

(b)

Fig. 5: Overview of trials conducted at Fire Service College.

an autonomous information theoretic mission, where an area was drawn on a map for the UAV to search within, guided by the information theoretic search algorithm that was assessed in Section V.

\section{A. Manual flight}

The first trial conducted was a manual flight around the oil rig. This trial was used as a visual inspection of the rig in addition to data collection using the onboard PID sensor. The photo in the top of Fig. 5b is an example snapshot of the footage during the initial visual survey. The flight is summarised in Fig. 6. After the initial loop of the rig done for visual surveying purposes the UAV returned to a downwind position where some initial detections had been made from the onboard PID sensor. The UAV flew crosswind near the downwind edge of the rig a few times to collect additional sensor data. The UAV was piloted from a position roughly $150 \mathrm{~m}$ North of the oil rig and it was not flown any closer to avoid a collision. The figure shows the final estimate of the source position given by the black cross, which fell approximately $3.6 \mathrm{~m}$ from the true position given by the blue circle. The heatmap in the figure is a forward prediction of the HAZMAT plume. This was generated by sampling from the posterior distributions of the source parameters, producing multiple forward runs with the dispersion model and averaging the outputs.

\section{B. Predefined flight}

The next demonstration was an autonomous waypoint mission around the car crash zone from Fig. 5. In a similar manner to the manual flight the data collected by the onboard PID detector was used to estimate the parameters of the source. The trial is summarised in Fig. 7. The final estimate of the source position was approximately $4.2 \mathrm{~m}$ from the true location of the source.

\section{Information theoretic flight}

Finally, the information theoretic flight that was assessed in experiments in Section V was demonstrated in the car crash vignette. The trial is summarised in Fig. 8. The search was terminated early in this test to avoid the UAV colliding with obstacles near the crash site. Despite this, the source was localised to within $4.5 \mathrm{~m}$ of its true position.

\section{Discussion}

The system performed well in the uncontrolled, realistic demonstration environments set out by the potential end users. In the manual tests it was useful for the end users to visually explore the hazardous environment using the onboard camera of the UAV. It enabled the pilot to fly downwind of suspicious looking objects to monitor for HAZMAT emissions. It would be interesting to achieve this functionality autonomously within the information theoretic planning algorithm by integrating computer vision algorithms into the estimation and planning framework, which has recently been explored in [28].

The pre-planned mission benefited from the increase in the number of spatio-temporal measurements of the HAZMAT which could enable airborne concentration distribution mapping algorithms to run in parallel to STE. The major drawback was the inability of the method to adjust its path in response to new information.

The information theoretic search performed well despite being in an untested larger scale environment with a different release source. However, it should be possible to estimate the source term much more quickly if the system also considers measurements whilst moving. One interesting component that was identified as useful would be the ability to continuously monitor the area once the source is identified, in order to refine the estimates and the source term and HAZMAT spread. Potentially linking the forward model based estimate of the plume from the source parameters with Gas Distribution Mapping (GDM) algorithms such as [5]-[7].

There were two key areas that were identified where the system is in need of further work: firstly, more advanced path 


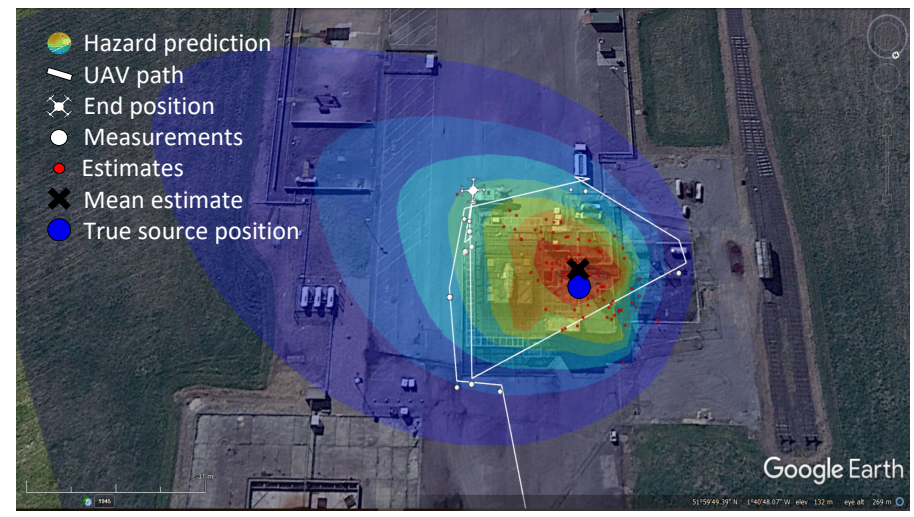

Fig. 6: Manual flight around a mock up oil rig with a suspected HAZMAT release.

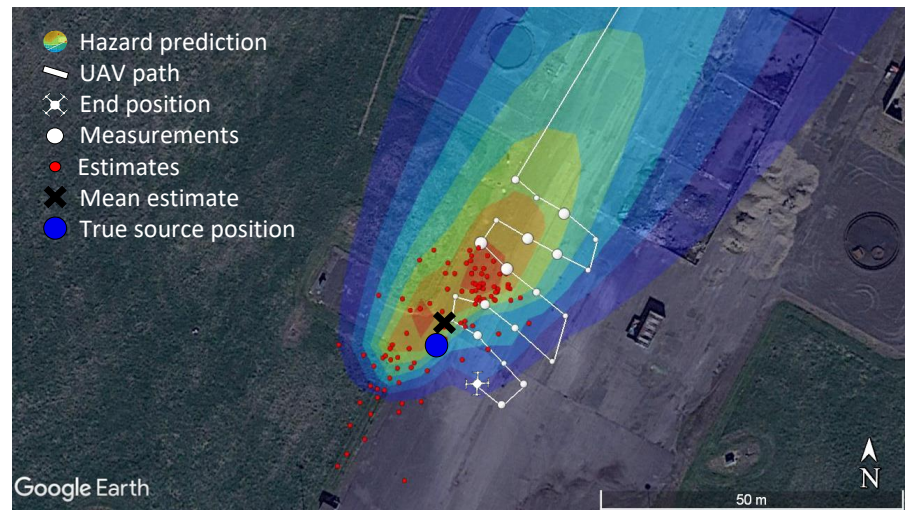

Fig. 7: Sweep search flight around a car crash incident with a suspected HAZMAT release.

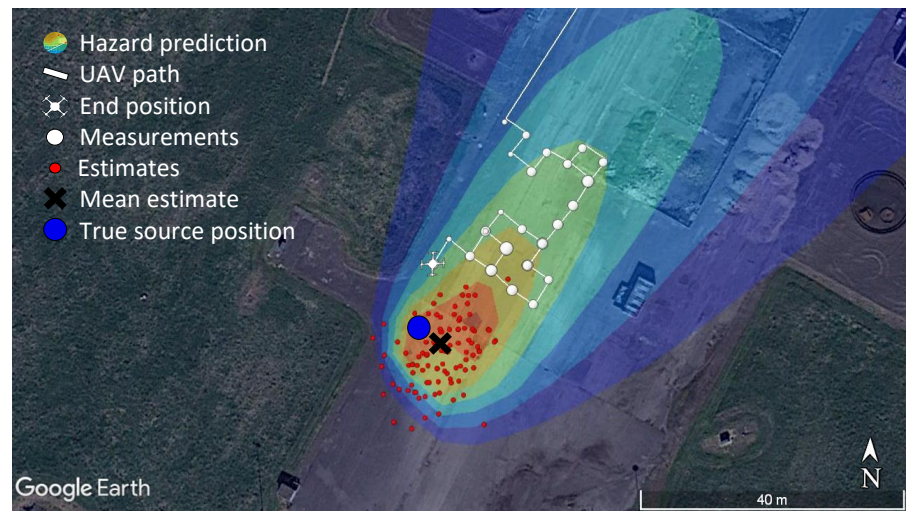

Fig. 8: Information theoretic search around a car crash incident with a suspected HAZMAT release.

planning and execution algorithms are critical when leaving the plain environment of the experiments in Section V, which will make the information theoretic search applicable to a larger range of scenarios; and secondly, it will be valuable to take into account global and local variations in the wind field both spatially and temporally throughout the mission, this will make the system more robust and accurate.

More advanced path planning and execution methods are needed to take into account obstacles in the environment. This could potentially be solved by replacing the path generator aspect of the system with Rapidly exploring Random Trees (RRT). It would also be useful to consider the effects of obstacles on the plume itself, either through a more accurate, higher fidelity dispersion model, which could be computationally expensive, or through modification of the likelihood function in the inference engine to take into account the uncertainty caused by an obstacle on the model that is used. In this online information gathering task, it will be important to consider computational complexity of the chosen solution.

Another important step to add robustness to the system will be to take into account variations in the wind field. During the demonstrations varying wind would have an adverse effect on the system. This could be caused by changes in the wind direction over time or by differences in the local wind at the meteorological station and at the HAZMAT source; exacerbated by a non-plain environment. Measuring the wind 
onboard the UAV, as in [27] and then incorporating it into the inference algorithm would benefit the system greatly. The variable wind will also require replacing the steady state dispersion model used for inference in this article with a time variant model.

\section{CONCLUSIONS}

This article has proposed a framework for information theoretic hazardous source search and reconstruction. Each component of the framework is extendible to improve the performance of the system or expand its application. The system is verified in experiments in an outdoor open field and then demonstrated at the Fire Service College, UK, in realistic scenarios developed by potential end users from the first responder community. The experiments showed the benefits of using the online information theoretic path planner over a predefined path from earlier work by the authors. The demonstrations showed the versatility of the system and confirmed important areas that are in need of further research.

\section{ACKNOWLEDGEMENT}

This project was jointly funded by the UK Ministry of Defence and the UK Home Office via the Defence and Security Accelerator under project number ACC500113.

The authors would like to acknowledge the kind assistance of the Dstl Minerva trials team and the staff of Fire Service College, Moreton-in-Marsh, Gloucestershire, UK, without whom the demonstrations would not have been possible.

\section{REFERENCES}

[1] M. Hutchinson, H. Oh, and W.-H. Chen, "A review of source term estimation methods for atmospheric dispersion events using static or mobile sensors," Information Fusion, vol. 36, pp. 130-148, 2017.

[2] M. Hutchinson, C. Liu, and W.-H. Chen, "Source term estimation of a hazardous airborne release using an unmanned aerial vehicle," Journal of Field Robotics, vol. 36, no. 4, pp. 797-817, 2019.

[3] M. Hutchinson, H. Oh, and W.-H. Chen, "Adaptive bayesian sensor motion planning for hazardous source term reconstruction," IFACPapersOnLine, vol. 50, no. 1, pp. 2812-2817, 2017.

[4] M. Hutchinson, C. Liu, and W.-H. Chen, "Information-based search for an atmospheric release using a mobile robot: Algorithm and experiments," IEEE Transactions on Control Systems Technology, no. 99, pp. $1-15,2018$.

[5] A. J. Lilienthal, M. Reggente, M. Trincavelli, J. L. Blanco, and J. Gonzalez, "A statistical approach to gas distribution modelling with mobile robots-the kernel dm+ v algorithm," in Intelligent Robots and Systems, 2009. IROS 2009. IEEE/RSJ International Conference on. IEEE, 2009, pp. $570-576$.

[6] C. Stachniss, C. Plagemann, and A. J. Lilienthal, "Learning gas distribution models using sparse gaussian process mixtures," Autonomous Robots, vol. 26, no. 2-3, pp. 187-202, 2009.

[7] J. G. Monroy, J.-L. Blanco, and J. Gonzalez-Jimenez, "Time-variant gas distribution mapping with obstacle information," Autonomous Robots, vol. 40 , no. 1 , pp. 1-16, 2016.

[8] G. Kowadlo and R. A. Russell, "Robot odor localization: a taxonomy and survey," The International Journal of Robotics Research, vol. 27, no. 8, pp. 869-894, 2008 .

[9] S. Qiu, B. Chen, R. Wang, Z. Zhu, Y. Wang, and X. Qiu, "Atmospheric dispersion prediction and source estimation of hazardous gas using artificial neural network, particle swarm optimization and expectation maximization," Atmospheric Environment, vol. 178, pp. 158 - 163, 2018

[10] Y. Wang, H. Huang, L. Huang, and B. Ristic, "Evaluation of bayesian source estimation methods with prairie grass observations and gaussian plume model: A comparison of likelihood functions and distance measures," Atmospheric Environment, vol. 152, pp. 519 - 530, 2017.
[11] C. D. Argyropoulos, S. Elkhalifa, E. Fthenou, G. C. Efthimiou, S. Andronopoulos, A. Venetsanos, I. V. Kovalets, and K. E. Kakosimos, "Source reconstruction of airborne toxics based on acute health effects information," Scientific reports, vol. 8, no. 1, p. 5596, 2018.

[12] B. Ristic, M. Morelande, and A. Gunatilaka, "Information driven search for point sources of gamma radiation," Signal Processing, vol. 90, no. 4, pp. 1225-1239, 2010

[13] C. Cadena, L. Carlone, H. Carrillo, Y. Latif, D. Scaramuzza, J. Neira, I. Reid, and J. J. Leonard, "Past, present, and future of simultaneous localization and mapping: Toward the robust-perception age," IEEE Transactions on robotics, vol. 32, no. 6, pp. 1309-1332, 2016.

[14] J. Tisdale, Z. Kim, and J. K. Hedrick, "Autonomous uav path planning and estimation," IEEE Robotics \& Automation Magazine, vol. 16, no. 2, pp. 35-42, 2009.

[15] D. Levine, B. Luders, and J. How, "Information-rich path planning with general constraints using rapidly-exploring random trees," in AIAA Infotech@Aerospace 2010,2010, p. 3360.

[16] O. M. Cliff, D. L. Saunders, and R. Fitch, "Robotic ecology: Tracking small dynamic animals with an autonomous aerial vehicle," Science Robotics, vol. 3, no. 23, p. eaat8409, 2018.

[17] G. A. Hollinger, B. Englot, F. S. Hover, U. Mitra, and G. S. Sukhatme, "Active planning for underwater inspection and the benefit of adaptivity," The International Journal of Robotics Research, vol. 32, no. 1, pp. 3-18, 2013.

[18] P. P. Neumann, V. Hernandez Bennetts, A. J. Lilienthal, M. Bartholmai, and J. H. Schiller, "Gas source localization with a micro-drone using bio-inspired and particle filter-based algorithms," Advanced Robotics, vol. 27, no. 9, pp. 725-738, 2013.

[19] M. Vergassola, E. Villermaux, and B. I. Shraiman, "infotaxis as a strategy for searching without gradients," Nature, vol. 445, no. 7126 , pp. 406-409, 2007

[20] J. Adler, "Chemotaxis in bacteria," Science, vol. 153, no. 3737, pp. 708716, 1966

[21] A. Keats, E. Yee, and F.-S. Lien, "Bayesian inference for source determination with applications to a complex urban environment," Atmospheric environment, vol. 41, no. 3, pp. 465-479, 2007.

[22] G. C. Efthimiou, I. V. Kovalets, A. Venetsanos, S. Andronopoulos, C. D. Argyropoulos, and K. Kakosimos, "An optimized inverse modelling method for determining the location and strength of a point source releasing airborne material in urban environment," Atmospheric Environment, 2017.

[23] K. S. Rao, "Uncertainty analysis in atmospheric dispersion modeling," pure and applied geophysics, vol. 162, no. 10, pp. 1893-1917, Oct 2005.

[24] E. T. Jaynes, Probability theory: The logic of science. Cambridge university press, 2003.

[25] W. R. Gilks and C. Berzuini, "Following a moving targetmonte carlo inference for dynamic bayesian models," Journal of the Royal Statistical Society: Series B (Statistical Methodology), vol. 63, no. 1, pp. 127-146, 2001.

[26] D. V. Lindley, "On a measure of the information provided by an experiment," The Annals of Mathematical Statistics, pp. 986-1005, 1956.

[27] P. P. Neumann, S. Asadi, A. J. Lilienthal, M. Bartholmai, and J. H. Schiller, "Autonomous gas-sensitive microdrone: Wind vector estimation and gas distribution mapping," IEEE robotics \& automation magazine, vol. 19, no. 1, pp. 50-61, 2012.

[28] J. Monroy, J.-R. Ruiz-Sarmiento, F.-A. Moreno, F. Melendez-Fernandez, C. Galindo, and J. Gonzalez-Jimenez, "A semantic-based gas source localization with a mobile robot combining vision and chemical sensing," Sensors, vol. 18, no. 12, p. 4174, 2018. 